\title{
QUADRATIC POLYNOMIALS WHICH HAVE A HIGH DENSITY OF PRIME VALUES
}

\author{
G. W. FUNG AND H. C. WILLIAMS
}

\begin{abstract}
The University of Manitoba Sieve Unit is used to find several values of $A(>0)$ such that the quadratic polynomial $x^{2}+x+A$ will have a large asymptotic density of prime values. The Hardy-Littlewood constants which characterize this density are also evaluated.
\end{abstract}

\section{INTRODUCTION}

Let $f_{A}(x)=x^{2}+x+A \quad(A \in \mathbf{Z}, A>0)$ and let $P_{A}(n)$ represent the number of prime values assumed by $f_{A}(x)$ for $x=0,1, \ldots, n$. In 1772 Euler discovered that $P_{41}(39)=40$. Indeed, the polynomial $x^{2}+x+41$ is well known to all students of number theory because of this remarkable property. Consider, however, the much less famous polynomial $x^{2}+x+27941$ discovered, according to Karst [4], by Beeger in 1938. Here $P_{27941}(39)=30$; but, $P_{27941}(1000000)=$ 286128 , whereas $P_{41}(1000000)=261080$. It appears, then, that while $f_{41}(x)$ starts off very well in the production of primes, the rather more modest $f_{27941}(x)$ begins to better its famous rival as the values of $x$ become large.

This phenomenon could have been predicted from Hardy and Littlewood's [3] Conjecture $F$. For the case of polynomials of the form $f_{A}(x)$ this conjecture can be given as

$$
P_{A}(n) \sim C(D) L_{A}(n),
$$

where $D=1-4 A, L_{A}(n)=2 \int_{0}^{n} d x / \log f_{A}(x)$, and

$$
C(D)=\prod_{p \geq 3}(1-(D / p) /(p-1)) .
$$

The product in (1.2) is taken over all the odd primes $p$, and by $(\cdot / p)$ we denote the Legendre symbol. Shanks [14] has computed $C(-163)=3.3197732$ and $C(-111763)=3.6319998$. Thus, on the basis of Conjecture $F$ one would expect that for sufficiently large values of $n, P_{27941}(n)$ would exceed $P_{41}(n)$,

Received March 10, 1988; revised April 24, 1989.

1980 Mathematics Subject Classification (1985 Revision). Primary 11N32, 11 Y 60,11 Y 40.

The second author's research was supported by NSERC of Canada Grant \#A7649. 
and this is what we have observed. Indeed, to five significant figures

$$
\begin{gathered}
P_{41}(100000) / L_{41}(1000000)=3.3203, \\
P_{27941}(1000000) / L_{27941}(1000000)=3.6397,
\end{gathered}
$$

both of which are quite close to their respective $C(D)$-values.

The purpose of this note is to find other polynomials $f_{A}(x)$ which have a high asymptotic density of prime values. We will do this by determining those values of $D$ for which the Hardy-Littlewood constant $C(D)$ should be large, and then evaluating $C(D)$ to eight significant figures. If Conjecture $F$ is true, then the corresponding values of $A$ should provide us with the desired polynomials. We point out here that all previous numerical tests of Conjecture $F$ have tended to confirm its truth (see Shanks [9-11]).

\section{StRategy FOR FINDING VAlues of $D$}

We first note that since we want $f_{A}(x)$ to assume prime values, then $A$ must be odd; hence, $-D=4 A-1 \equiv 3(\bmod 8)$. In order to maximize the asymptotic value of $P_{A}(n)$, we can maximize $C(D)$. According to (1.2) this means that we would want $(D / p)=-1$ for as many of the small primes $p$ as possible. As noted by Lehmer [5], we can also look at this from the point of view of restricting the number of possible small prime divisors of $f_{A}(x)$. If $(D / p)=-1$, then $p$ cannot divide $f_{A}(x)$ for any value of $x$; thus, if $(D / p)=-1$ for many small primes $p$, then the composite values that $f_{A}(x)$ can assume are considerably restricted. It follows that $f_{A}(x)$ should frequently be a prime.

If we let $N_{r}$ denote the least positive integer such that $N_{r} \equiv 3(\bmod 8)$ and $\left(N_{r} / p\right)=-(-1 / p)$ for all odd primes $p \leq p_{r}$, where $p_{r}$ is the $r$ th prime, then $-N_{r}$ should be a good candidate for the kind of $D$-value that we are seeking. This was the strategy used in [5] to find values for $D$. In Table 2.1 we give all the values of $N_{r}$ up to $r=42$.

The values of $N_{r}$ for $r \leq 28$ are given in [5]; the values of $N_{r}$ for $r \leq 38$ are given in Lehmer, Lehmer, and Shanks [6]; and the values of $N_{r}$ for $r=39$, $40,41,42$ were found by D. H. Lehmer but have not been previously published, except for $N_{42}$ which appears in Shanks [15].

To find all these values of $N_{r}$, Lehmer made use of mechanized or electronic number sieving devices. Such specialized machines are small, fast, and much less expensive than general purpose computers. In the production of the numbers presented in this paper we made use of the University of Manitoba Sieve Unit (UMSU) [8]. This device solves systems of linear congruences at a trial rate of 133,000,000 numbers per second. Thus, in about three months of continuous use, we were able to examine numbers up to $10^{15}$. 
TABLE 2.1

\begin{tabular}{r|r|r||r|r|r}
\hline$r$ & $p_{r}$ & \multicolumn{1}{|c|}{$N_{r}$} & $r$ & $p_{r}$ & \multicolumn{1}{|c}{$N_{r}$} \\
\hline & & & & & \\
2 & 3 & 19 & 23 & 83 & 114148483 \\
3 & 5 & 43 & 24 & 89 & 269497867 \\
4 & 7 & 43 & 25 & 97 & 269497867 \\
5 & 11 & 67 & 26 & 101 & 269497867 \\
6 & 13 & 67 & 27 & 103 & 269497867 \\
7 & 17 & 163 & 28 & 107 & 585811843 \\
8 & 19 & 163 & 29 & 109 & 52947440683 \\
9 & 23 & 163 & 30 & 113 & 52947440683 \\
10 & 29 & 163 & 31 & 127 & 71837718283 \\
11 & 31 & 163 & 32 & 131 & 229565917267 \\
12 & 37 & 163 & 33 & 137 & 229565917267 \\
13 & 41 & 77683 & 34 & 139 & 575528148427 \\
14 & 43 & 77683 & 35 & 149 & 1432817816347 \\
15 & 47 & 1333963 & 36 & 151 & 1432817816347 \\
16 & 53 & 2404147 & 37 & 157 & 1432817816347 \\
17 & 59 & 2404147 & 38 & 163 & 1432817816347 \\
18 & 61 & 20950603 & 39 & 167 & 6778817202523 \\
19 & 67 & 36254563 & 40 & 173 & 16501779755323 \\
20 & 71 & 51599563 & 41 & 179 & 30059924764123 \\
21 & 73 & 96295483 & 42 & 181 & 30059924764123 \\
22 & 79 & 96295483 & & & \\
\hline
\end{tabular}

Note that Beeger's number, 111763 , is not in Table 2.1, yet it has a better $C(D)$-value than $C(-77683)=3.3003388<C(-163) .{ }^{1}$ If we put $N_{r, 1}=N_{r}$ above and define $N_{r, i}(i>1)$ as the least integer greater than $N_{r, i-1}$ such that $N_{r, i} \equiv 3(\bmod 8)$ and $\left(N_{r, i} / p\right)=-1$ for all odd primes $p \leq p_{r}$, then $111763=N_{43,2}$. Thus, instead of attempting simply to tabulate more $N_{r}$ values than those given in Table 2.1, we used UMSU to compute $N_{r, i}$ for $r \leq 40$ and $i \leq 10$. For $r=41$ we let UMSU continue to find values of $N_{r, i}$ until these values exceeded $10^{15}$. We were thus able to find all $N_{41, i}$ for $i \leq 15$. Having these candidates for $D$, the next problem is to determine those that yield the largest $C(D)$ values. Unfortunately, the product (1.2) converges very slowly; hence, we must develop an alternative method of computing $C(D)$, especially for large values of $D$.

\section{Computation of $C(D)$}

Efficient methods for evaluating $C(D)$ have been developed by Shanks [10, $11,14]$. In $[10,11]$ he discovered a method of finding $C(D)$ which appears to work well when $D$ is fairly small, and in [14] he provided a method of determining $C(D)$ to high accuracy, which will work when $D$ is larger. Indeed, he provides values for $C(-163), C(-77683), C(-111763), C(-289963)$, $C(-991027)$, the latter value being 4.1237067 , the largest $C$-value known

\footnotetext{
'Shanks [14] gets $3.2999354 \ldots$ for $\left({ }^{\prime}(-77683)\right.$; however, we evaluated this number in two different ways and still got 3.3003388. Thus we feel that some minor error crept into Shanks' evaluation of $C(-77683)$. Our results agree with all of Shanks' other evaluations.
} 
until now. If we put

$$
L(s, \chi)=\sum_{n=1}^{\infty} \chi(n) n^{-s}=\prod_{p}\left(1-\chi(p) / p^{s}\right)^{-1},
$$

where $\chi(n)=(D / n)$ and $(\cdot / n)$ is the Kronecker symbol, then all of these methods require that $L(s, \chi)$ be computed for various values of $s$. If $h$ is the class number of $\mathscr{Q}(\sqrt{D})$, the value of $L(s, \chi)$ can be determined fairly readily when the values of $h$ Epstein zeta functions are known. As Shanks [14] can rapidly evaluate these Epstein zeta functions to high accuracy, he can then accurately compute $C(D)$. However, if $h$ is large, this method can be quite slow. It is, however, the best method to use when $C(D)$ is needed to great accuracy. Also, this technique, unlike the one we will discuss below is unconditional.

As it was necessary for us to calculate $C(D)$ for many $D$-values, some of which were very large, we needed to develop a faster method to compute $C(D)$. We also assumed that evaluating $C(D)$ to eight significant figures would be adequate for the purposes of this note.

We first point out that if we use an idea in [10], it is a simple matter to show that

$$
\frac{C(D) L(1, \chi) L(2, \chi)}{\zeta(4)}=\frac{1}{2} \prod_{p \mid D}\left(1-\frac{1}{p^{4}}\right) \prod_{q \geq 3}\left(1-\frac{2}{q(q-1)^{2}}\right),
$$

where the first product on the right is taken over all the primes $p$ which divide $D$ and the second is taken over all primes $q$ such that $(D / q)=1$. Since $\zeta(4)=\pi^{4} / 90$ and

$$
\pi h / \sqrt{|D|}=L(1, \chi)
$$

we get

$$
C(D)=\frac{\pi^{3} \sqrt{|D|}}{180 h} \cdot \frac{1}{L(2, \chi)} \cdot \prod_{p \mid D}\left(1-\frac{1}{p^{4}}\right) \prod_{q \geq 3}\left(1-\frac{2}{q(q-1)^{2}}\right) .
$$

Put

$$
\begin{array}{ll}
F_{1}(Q)=\prod_{p \leq Q} p /(p-\chi(p)), & T_{1}(Q)=\prod_{p>Q} p /(p-\chi(p)), \\
F_{2}(Q)=\prod_{p \leq Q} p^{2} /\left(p^{2}-\chi(p)\right), & T_{2}(Q)=\prod_{p>Q} p^{2} /\left(p^{2}-\chi(p)\right), \\
F_{3}(Q)=\prod_{q \leq Q}\left(1-\frac{2}{q(q-1)^{2}}\right), & T_{3}(Q)=\prod_{q>Q}\left(1-\frac{2}{q(q-1)^{2}}\right) .
\end{array}
$$

By examining (3.1) and (3.2), we see that two problems arise in computing $C(D)$ : (1) evaluate $L(1, \chi)$ to sufficient accuracy to determine $h,(2)$ find $Q$ 
such that

$$
C(D) \approx \frac{\pi^{3} \sqrt{|D|}}{180 h} \frac{F_{3}(Q)}{F_{2}(Q)} \prod_{p \mid D}\left(1-\frac{1}{p^{4}}\right)
$$

approximates $C(D)$ to eight significant figures.

It is a simple matter to show that

$$
\left|\log T_{2}(Q)\right|+\left|\log T_{3}(Q)\right|=\left|\sum_{p>Q} \chi(p) / p^{2}\right|+\delta_{1},
$$

where $\left|\delta_{1}\right|<2 / Q^{2} \quad(Q>10)$. Also,

$$
\left|\log T_{1}(Q)\right|=\left|\sum_{p>Q} \chi(p) / p\right|+\delta_{2},
$$

where $\left|\delta_{2}\right|<1 / Q$. If we set

$$
B(Q)=\log |D|\left(\frac{1}{\pi \log Q}+\frac{5 \cdot 3}{(\log Q)^{2}}\right)+\frac{4}{\log Q}+\frac{1}{\pi},
$$

then by using the method of Cornell and Washington [2], we get

$$
\left|\log T_{2}(Q)\right|+\left|\log T_{3}(Q)\right|<B(Q)(8+13 \log Q) /\left(9 Q^{3 / 2}\right)+2 / Q^{2}
$$

and

$$
\left|\log T_{1}(Q)\right|<B(Q)(4+3 \log Q) / Q^{1 / 2}+1 / Q=A(Q) .
$$

It is important to note that the proof of these inequalities requires the truth of the Riemann Hypothesis on $L(s, \chi)$. Thus, the method that we develop here for finding $C(D)$ is contingent on the Extended Riemann Hypothesis.

Now if

$$
\left|\log T_{2}(Q)\right|+\left|\log T_{3}(Q)\right|<b,
$$

then (3.3) will approximate $C(D)$ to $r$ significant figures if $b<\log ((1+$ $\sqrt{1+4 k}) / 2$ ), where $k=10^{1-r} / 2$. Hence, by (3.4), if $D \approx 10^{15}$, then $Q=10^{6}$ in (3.3) will yield $C(D)$ to eight figures. Of course, for smaller values of $D$, smaller values of $Q$ can be used in (3.3). To test this, we evaluated (3.3) for the largest $D$-values we found with $Q=10^{6}$ and $Q=5 \cdot 10^{6}$. In every case, both computations agreed to eight significant figures.

There remains the problem of determining $h$. For this problem we used a modification of the idea of Lenstra [7]. If, for a fixed $Q$, we put

$$
\begin{aligned}
\pi B_{1} & =\sqrt{|D|} F_{1}(Q) \exp (A(Q)), \quad \tilde{h}=\operatorname{Ne}\left(\sqrt{|D|} F_{1}(Q) / \pi\right), \\
k & =\sqrt{|D|} F_{1}(Q) / \pi-\tilde{h}, \quad B_{2}=|k|+B_{1}-\sqrt{|D|} F_{1}(Q) / \pi,
\end{aligned}
$$

then by (3.1) and (3.5) we have

$$
h \leq B_{1}, \quad|\tilde{h}-h|<B_{2} .
$$


Here, by $\operatorname{Ne}(x)$ we denote the nearest integer to $x$. Now if we know a divisor $h_{1}$ of $h$ such that

$$
\tilde{h} / h_{1}-B_{2} / h_{1}-\left[B_{2} / h_{1}+\tilde{h} / h_{1}\right]>-1,
$$

then $h_{2}=\left[B_{2} / h_{1}+\tilde{h} / h_{1}\right]$ is the only integer in the interval $I$ given by

$$
\tilde{h} / h_{1}-B_{2} / h_{1}<x \leq\left[B_{2} / h_{1}+\tilde{h} / h_{1}\right] \text {. }
$$

Since $\left|h / h_{1}-\tilde{h} / h_{1}\right|<B_{2} / h_{1}$, we see that $h / h_{1}$ must be in $I$. It follows that $h=h_{1} h_{2}$ when (3.6) holds.

If (3.6) does not hold for $h_{1}=1$, we can use the baby step-giant step method of Shanks [12] to find a divisor $h_{1}$ of $h$ such that $h_{1}>1$. In fact, since most of the class groups of $Q(\sqrt{D})$ are cyclic or close to it (see Cohen and Lenstra [1]), this technique rapidly provides a value of $h_{1}$ which is close to $h$ in value; hence, $(3.6)$ is usually satisfied very quickly.

\section{NUMERICAL RESUltS}

The method described in $\S 3$ was programmed in FORTRAN with some assembly language subroutines and run on an Amdahl 5870 computer. For a given $D$, a value of $Q$ was determined which would guarantee eight figures of accuracy for $C(D)$ by $(3.3)$. The values of $F_{1}(Q), F_{2}(Q), F_{3}(Q)$ were evaluated simultaneously in double precision by the assembly language subroutines.

The $C(D)$-values for the 192 numbers found by UMSU were computed in a total of about three CPU minutes. Denote by $q(D)$ the least prime such that $(D / q(D)) \neq-1$. In Table 4.1 we give all the numbers $D$ found by UMSU with $q(D) \geq 163$. We also provide the corresponding values of $h, C(D)$, and $q(D)$.

Notice that 110587910656507 allows us to extend Table 2.1. In fact, this number is $N_{43}, N_{44}$, and $N_{45}$. It is rather unfortunate that $\left(-N_{43} / 199\right)=1$ because $\left(-N_{43} / p\right)=-1$ for $p=211,223,227,229$, and 223. Thus, if it were not for the value of the Legendre symbol for 199 we would have $N_{51}=N_{43}$. As it is, the best that we can say here is that $N_{46}>10^{15}$.

In Table 4.2 we give those values of $D$ from among the 192 such that $C(D)>$ $C\left(D^{\prime}\right)$ for all the $D^{\prime}$, among the 192 , which are less than $D$. We also give the corresponding value of $P_{A}(1000000)$ and $P_{A}(1000000) / L_{A}(1000000)$ (written as $P(1000000)$ and $P / L$, respectively), where $A=(1-D) / 4$. Also, since the $C(D)$-values are roughly inversely proportional to the respective $L(1, \chi)$-values, we provide these values as $L(D)=L_{-D}(1)=\frac{3}{2} L(1, \chi)$ in order to permit comparison with the results of Shanks [13]. Notice that $P_{A}(1000000) / L_{A}(1000000)$ and $C(D)$ are quite close, providing yet further confirmation of Conjecture $F$.

Let $D^{\prime}$ denote the last $D$-value in Table $4.2\left(D^{\prime}=-531 \ldots\right)$. As we would expect, for $D=-N_{43}$ we get a quite large $C(D)$-value. What appears, at first, to be somewhat remarkable is that this $C(D)$-value is less than $C\left(D^{\prime}\right)$; however, even though $q\left(-N_{43}\right)>q\left(D^{\prime}\right)$, beyond $q\left(D^{\prime}\right)$ we get a higher density of nonresidues for $D^{\prime}$ than for $-N_{43}$, hence the larger $C(D)$-value for $D^{\prime}$. 
TABLE 4.1

\begin{tabular}{rrcr}
\hline \multicolumn{1}{c}{$h$} & \multicolumn{1}{c}{$C$} & $C(D)$ & $q(D)$ \\
\hline-1432817816347 & 70877 & 4.4163429 & 167 \\
-5066580103267 & 131930 & 4.4616823 & 163 \\
-6626709638707 & 148069 & 4.5468709 & 163 \\
-6778817202523 & 149460 & 4.5565681 & 173 \\
-8547099746707 & 176959 & 4.3197166 & 163 \\
-8903633500507 & 168563 & 4.6296715 & 163 \\
-9275311526083 & 179187 & 4.4443528 & 163 \\
-15159061903507 & 225866 & 4.5075910 & 163 \\
-16501779755323 & 223574 & 4.7524812 & 179 \\
-17542900082563 & 240468 & 4.5549908 & 163 \\
-30059924764123 & 296475 & 4.8379057 & 191 \\
-37221595794667 & 328170 & 4.8634109 & 179 \\
-50923056589267 & 423835 & 4.4030138 & 179 \\
-58212094833523 & 427986 & 4.6627278 & 167 \\
-58369246601803 & 429990 & 4.6472033 & 173 \\
-64279195020307 & 454960 & 4.6086679 & 173 \\
-65569185073723 & 444070 & 4.7692730 & 167 \\
-69298004348827 & 474064 & 4.5926958 & 173 \\
-74210430269347 & 454842 & 4.9548401 & 181 \\
-82973459224363 & 530640 & 4.4889020 & 173 \\
-87934318851787 & 528770 & 4.6380011 & 181 \\
-88795060352923 & 519882 & 4.7412369 & 179 \\
-110587910656507 & 553436 & 4.9711959 & 199 \\
-126620398458283 & 640888 & 4.5916614 & 179 \\
-138411891537187 & 655794 & 4.6925243 & 179 \\
-307568240581123 & 949638 & 4.8308250 & 181 \\
-378486993318883 & 1082408 & 4.7014317 & 193 \\
-404210888356867 & 1123425 & 4.6808868 & 191 \\
-414286790833987 & 1158810 & 4.5940080 & 181 \\
-531497118115723 & 1185668 & 5.0870883 & 181 \\
-696687486054883 & 1410630 & 4.8947820 & 191 \\
-772147706149747 & 1529796 & 4.7510872 & 181 \\
-792933985668883 & 1578348 & 4.6664594 & 181 \\
-799705726392763 & 1554144 & 4.7590191 & 181 \\
-850229380873387 & 1596966 & 4.7756941 & 191 \\
-998727466696243 & 1789388 & 4.6191771 & 181 \\
\hline & & & \\
\hline
\end{tabular}

To further illustrate this phenomenon, we give in Table 4.3 some more $C(D)$ values. The $D$-values in this table are taken from the latter part of Shanks' table of Lochamps (Table 3) in [13]. We have only selected those $D$-values which are not already in Table 4.2 and are congruent to 5 modulo 8 . We have also reproduced the $L(D)$-values given in Shanks' table.

Notice that for $D=-991027$ and $D=-3416131987$ we get larger $C(D)$ values than those given in Table 4.2 for $D$-values of comparable size. Thus, there may be more numbers $<10^{15}$ with $C(D)$-values in excess of $C\left(-N_{43}\right)$. What we can say here is that if Conjecture $F$ holds, then

$$
x^{2}+x+132874279528931
$$


TABLE 4.2

\begin{tabular}{rcccc}
\hline \multicolumn{1}{c}{$D$} & $P(1000000)$ & $C(D)$ & $P / L$ & $L(D)$ \\
\hline-163 & 261080 & 3.3197732 & 3.3203421 & 0.3691028 \\
-85507 & 272102 & 3.4643422 & 3.4612190 & 0.3545382 \\
-111763 & 286128 & 3.6319998 & 3.6396821 & 0.3383011 \\
-222643 & 293169 & 3.7289570 & 3.7293962 & 0.3295722 \\
-1333963 & 300001 & 3.8123997 & 3.8169182 & 0.3223267 \\
-9471067 & 312436 & 3.9760501 & 3.9764927 & 0.3093093 \\
-10560643 & 315542 & 4.0194873 & 4.0161335 & 0.3059697 \\
-60408307 & 318250 & 4.0501092 & 4.0531570 & 0.3037600 \\
-171583003 & 320126 & 4.0815068 & 4.0796515 & 0.3014727 \\
-269497867 & 322488 & 4.1092157 & 4.1112637 & 0.2996843 \\
-398158363 & 325782 & 4.1579113 & 4.1548155 & 0.2961493 \\
-643338763 & 335224 & 4.2716019 & 4.2775772 & 0.2883454 \\
-1408126003 & 334712 & 4.2771747 & 4.2759778 & 0.2879549 \\
-1595514187 & 341572 & 4.3616794 & 4.3645752 & 0.2824327 \\
-4067175907 & 346057 & 4.4324788 & 4.4309683 & 0.2779060 \\
-71837718283 & 354875 & 4.6097143 & 4.6090901 & 0.2673146 \\
-85702502803 & 361841 & 4.7073044 & 4.7067227 & 0.2617208 \\
-16501779755323 & 326605 & 4.7524812 & 4.7559512 & 0.2593564 \\
-30059924764123 & 326392 & 4.8379057 & 4.8453809 & 0.2548210 \\
-37221595794667 & 325086 & 4.8634109 & 4.8594354 & 0.2534793 \\
-74210430269347 & 323289 & 4.9548401 & 4.9413604 & 0.2488108 \\
-110587910656507 & 321488 & 4.9711959 & 4.9770300 & 0.2480017 \\
-531497118115723 & 312975 & 5.0870883 & 5.0894316 & 0.2423560 \\
\hline
\end{tabular}

TABLE 4.3

\begin{tabular}{rcccc}
\hline \multicolumn{1}{c}{$D$} & $P(1000000)$ & $C(D)$ & $P / L$ & $L(D)$ \\
\hline & & & & \\
-546067 & 297046 & 3.7775732 & 3.7789730 & .32523 \\
-991027 & 324001 & 4.1237067 & 4.1221307 & .29822 \\
-1970364883 & 339556 & 4.3367305 & 4.3405407 & .28398 \\
-2426489587 & 343914 & 4.4024373 & 4.3981264 & .27982 \\
-3416131987 & 353395 & 4.5247200 & 4.5229186 & .27227 \\
-8864190043 & 355373 & 4.5655590 & 4.5612380 & .26983 \\
\hline
\end{tabular}

is a quadratic polynomial which has a higher asymptotic density of prime values than any other such polynomial known to date.

\section{ACKNOWLEDGMENT}

The authors wish to thank D. H. Lehmer for making his unpublished work available to them. They also wish to thank Daniel Shanks for many helpful observations and references.

\section{BIBLIOGR.APHY}

1. H. Cohen and H. W. Lenstra, Jr., Heuristics on class groups of number fields, Number Theory (Noordwijkerhout, 1983). Lecture Notes in Math.. vol. 1068. Springer-Verlag. Berlin and New York, 1984, pp. 33-62. 
2. G. Cornell and L. C. Washington, Class numbers of cyclotomic fields, J. Number Theory 21 (1985), 260-274.

3. G. H. Hardy and J. E. Littlewood, Partitio numerorum III: On the expression of a number as a sum of primes, Acta Math. 44 (1923), 1-70.

4. E. Karst, The congruence $2^{p-1} \equiv 1\left(\bmod p^{2}\right)$ and quadratic forms with high density of primes, Elem. Math. 22 (1967), 85-88.

5. D. H. Lehmer, On the function of $x^{2}+x+A$, Sphinx 6 (1936), 212-214 and Sphinx 7 (1937), 40.

6. D. H. Lehmer, E. Lehmer, and D. Shanks, Integer sequences having prescribed quadratic character, Math. Comp. 24 (1970), 433-451.

7. H. W. Lenstra, Jr., On the calculation of regulators and class numbers of quadratic fields, London Math. Soc. Lecture Note Ser., vol. 56, Cambridge Univ. Press, Cambridge and New York, 1982, pp. 123-150.

8. C. D. Patterson and H. C. Williams, A report on the University of Manitoba Sieve Unit, Congr. Numer. 37 (1983), 85-98.

9. Daniel Shanks, A sieve method for factoring numbers of the form $n^{2}+1$, MTAC 13 (1959), 78-86.

10. On the conjecture of Hardy and Littlewood concerning the number of primes of the form $n^{2}+a$, Math. Comp. 14 (1960), 320-332.

11. __ Supplementary data and remarks concerning a Hardy-Littlewood conjecture, Math. Comp. 17 (1963), 188-193.

12. _ Class number, a theory of factorization and genera, Proc. Sympos. Pure Math., vol. 20 (1969 Institute on Number Theory), Amer. Math. Soc., Providence, R.I., 1971, pp. $415-440$.

13. __ Systematic examination of Littlewood's bounds on $L(1, \chi)$, Proc. Sympos. Pure Math., vol. 24, Amer. Math. Soc., Providence, R.I., 1973, pp. 267-283.

14. $\ldots$, Calculation and applications of Epstein zeta functions, Math. Comp. 29 (1975), 271 287.

15. __ A survey of quadratic, cubic and quartic algebraic number fields, Congr. Numer. 17 (1976), 15-42.

Department of Computer Science, University of Manitoba, Winnipeg, Manitoba, CANADA R3T 2N2 